'M Kakinda, 'I C Opio, '2J K B Matovu. 'Children's AIDS Fund, Kampala, Uganda, ${ }^{2}$ Makerere University School of Public Health, Kampala, Uganda

Background We implemented a facility-based intervention aimed at reducing the proportion of patients lost to follow-up at an outpatient HIV clinic in Jinja district, Eastern Uganda, over a period of 6 months (February-July 2012)

Methods The intervention was implemented with the aim of decreasing the proportion of patients lost to follow-up from $1 \%$ $(23 / 2328)$ at baseline to $0.25 \%$ by July 2012 . Simple and innovative strategies were introduced into the HIV clinic. These included retraining of clinic staffs on the importance of patients keeping their appointments; development of new messages on keeping appointments; retrieving patient files a day before the day of appointment; sending two text message reminders a day to the clinic; and actively following up patients who had not kept their appointments through home-visiting. At each follow-up visit, reasons for the patients' failure to keep appointment were noted and information on age, gender, CD4 count (captured from patient records) and duration in HIV care was obtained. Data were fed into an IQ Care programme and analysed using Microsoft Excel.

Results The proportion of patients lost to follow-up decreased from $1 \%(23 / 2328)$ at baseline to $0.4 \%(9 / 2528)$ in 6 months. Men, those with a CD4 $>350$, those aged $<30$ years and those in care for $<1$ year had the biggest decline in proportion of patients lost to follow-up compared to other patients.

Conclusion The proportion of patients lost to follow-up declined by $60 \%$ through use of simple and innovative strategies introduced in the HIV clinic.

\section{P6.058 UNIVERSAL HIV SCREENING OF INMATES IN ISRAELI PRISONS: SHOULD THE POLICY BE UPDATED?}

doi:10.1136/sextrans-2013-051184.1211

'Z Mor, ${ }^{2} \mathrm{H}$ Vider, ${ }^{3,4} \mid \mathrm{G}$ Grotto, ${ }^{5} \mathrm{D}$ Tischler Aurkin. ${ }^{1}$ Ministry of Health, Ramla, Israel; ${ }^{2}$ ssraeli prison Services, Ramla, Israel; ${ }^{3}$ Ministry of Health, Jerusalem, Israel; ${ }^{4} B e n$ Gurion University, Beer Sheva, Israel; ${ }^{5}$ sraeli Prison Services, Ramla, Israel

Background HIV rates among inmates are mostly higher than the general population. Israel is a relative low-HIV prevalence country (107:100,000 population, 2011 data). All criminal inmates incarcerated in prisons/gaols at the Israeli Prison Services (IPS) are routinely screened for HIV. This retrospective study evaluates the necessity of the current policy of universal HIV-testing policy.

Methods We reviewed all inmates diagnosed with HIV/AIDS upon incarceration in Israel between 2003 and 2010, and assessed their risk-behaviours and the date of diagnosis.

Results During the 8 years follow-up, 108,866 new criminal inmates were incarcerated in 31 correctional facilities, and it is estimated that $95 \%$ of those were tested for HIV. Of those, $201(0.2 \%)$ were tested positively with HIV, in a direct testing cost of 622,000 (US\$).

Of all $201 \mathrm{HIV}$-infected inmates, 118 (58.7\%) were intra-venous drug-users (IVDU), 55 (27.4\%) originated in high-prevalence countries, $13(6.5 \%)$ were men who have sex with men (MSM), 12 (6.0\%) were heterosexuals not originating in endemic country, $2(0.1 \%)$ the risk-group was undetermined and one $(0.5 \%)$ was infected vertically.

Of all 201 HIV-infected inmates, 157 (78.2\%) were diagnosed in the community, prior to their imprisonment, and were re-tested in prison; while $44(21.8 \%)$ were firstly diagnosed in prison. Of those 44 inmates, $25(56.8 \%)$ were IVDU, $13(29.5 \%)$ originated in endemic country, three $(9.1 \%)$ were MSM and in two $(4.5 \%)$ the risk-group was not determined.

Conclusion HIV-infection rate is prison is twice higher than the general population. The majority $(98.5 \%)$ of all inmates was diagnosed prior to their incarceration or had a key risk-behaviour expos- ing them to HIV. Therefore, questioning each new inmate upon incarceration about previous HIV-diagnosis, and targeted testing for other inmates who are IVDU, MSM or originating in endemic countries for HIV can detect almost all HIV-infected prisoners, presuming they respond reliably.

\section{P6.059 VERTICAL TRANSMISSION OF HIV LESS THAN HALF AMONG MOTHERS BELONGING TO MOTHER-SUPPORT GROUPS (MSG) COMPARED TO NON-MEMBER MOTHERS AT HEALTH CENTRES IN TIGRAY, ETHIOPIA}

doi:10.1136/sextrans-2013-051184.1212

'M Abraha, 'T A Kahsu, ${ }^{2} \mathrm{H}$ Godefay, 'E Konings. 'Management Sciences for Health, Addis Ababa, Ethiopia, ${ }^{2}$ Tigray Regional Health Bureau, Mekele, Ethiopia

Background Mother-support groups (MSGs) are used at hospitals and health centres in Ethiopia to increase uptake of and adherence to PMTCT and reduce vertical transmission of HIV. A study at Dessie Referral Hospital showed that mothers belonging to MSGs had lower vertical transmission rates. The impact on mothers seen at health centres is not known.

Methods Data on the outcome of MSGs on vertical transmission were collected from 15 health centres in Tigray between 2008 and 2011. Data sources included MSG registration log books, regional laboratory DNA/PCR results, and the ART enrollment register. We compared HIV status among babies born to mothers belonging to MSG and those whose mothers did not participate in MSG.

Results A total of 848 HIV-exposed infants (HEIs) were registered between 2008 and 2011. Among 240 HEIs born to mothers enrolled in MSG, $87.5 \%$ were tested for HIV and $5.2 \%$ were HIV-positive. Among the 608 HEIs whose mothers were not enrolled in MSGs, $44.7 \%$ were tested and $11.4 \%$ tested HIV-positive (OR $=0.43$ ).

Conclusions The odds of testing positive were 57\% lower among HEIs whose mother was enrolled in an MSG compared to those whose mother was not enrolled. The better outcome appears to be due to the support their mothers received from MSGs resulting in better PMTCT and adherence practises as well as high rates of institutional delivery. We are currently collecting more data on PMTCT regimen, HEI testing by age and place of delivery to verify this hypothesis.

\section{P6.060 PATIENT OR PROVIDER REFERRAL FOR CHLAMYDIA - WHAT IS THE COST AND IS IT WORTH IT? A COST COMPARISON OF ALTERNATIVE STRATEGIES}

doi:10.1136/sextrans-2013-051184.1213

${ }^{1}$ T Roberts, ${ }^{2 \mathrm{H}}$ Mistry, 'M Rossello-Roig, ${ }^{3} \mathrm{G}$ Rait, ${ }^{4} \mathrm{~J}$ Dodds, ${ }^{5} \mathrm{~S}$ Lanza, ${ }^{4} \mathrm{C} S$ Estcourt, ${ }^{6} \mathrm{M}$ Symonds, ${ }^{5} \mathrm{~J}$ A Cassell. 'University of Birmingham, Birmingham, UK; ${ }^{2}$ University of Warwick, Coventry, UK; ${ }^{3}$ University College London, London, UK; ${ }^{4}$ Queen Mary, University of London, London, UK; ${ }^{5}$ Brighton and Sussex Medical School, Brighton, UK; ${ }^{6}$ Barts Health NHS Trust, London, UK

Background Partner notification (PN) is an essential element of STI control. Typically partner notification has been supported by specialist health advisors based in GUM clinics, but recently the role has been extended to community based Chlamydia screening officers (including primary care). We aimed to explore and compare costs of various approaches to $\mathrm{PN}$ for Chlamydia in different settings.

Methods We compared costs of being offered one of five approaches to partner notification from the health service perspective:

1. Routine specialist clinic PN (patient referral including infection specific information, and advice that the sex partner should attend clinic for testing and treatment)

2. Accelerated Partner Therapy (APT Hotline): nurse initiated $\mathrm{PN}$ at the general practise followed by telephone assessment of sex partner by clinic-based nurse qualified health adviser; 